\title{
A PROBABILISTIC APPROACH FOR LONG-TERM FATIGUE ANALYSIS OF ONSHORE WIND TURBINE TOWER
}

\section{HAO BAI ${ }^{1}$, DIDIER LEMOSSE ${ }^{1}$, YOUNES AOUES ${ }^{1}$, JEAN-MARC CHERFILS $^{1}$ AND CHANGWU HUANG ${ }^{2}$}

${ }^{1}$ Laboratory of Mechanics of Normandy (LMN)

INSA Rouen Normandy, 76000 Rouen, France hao.bai@insa-rouen.fr

2 Department of Computer Science and Engineering Southern University of Science and Technology, Shenzhen 518055, China huangcw3@sustech.edu.cn

Key words: Reliability analysis, Cumulative fatigue damage, Wind turbine tower, Probabilistic fatigue analysis

\begin{abstract}
To address the fatigue damage induced by wind on the wind turbine tower, the present work introduces a novel probabilistic fatigue assessment framework. The idea is based on the deterministic fatigue approach combining with various statistical and probabilistic techniques. The proposed framework is applied to a Design Load Case (DLC) given in IEC 61400-1 standard using a reference wind turbine and carry out a probability distribution of cumulative fatigue damage on the cross-section of wind turbine tower under a turbulent wind condition.
\end{abstract}

\section{INTRODUCTION}

The random nature of the wind actions could cause unexpected fatigue damage and thus reduce the wind turbine's operating lifetime. With the increasing size of wind turbine tower, the fatigue damage induced by the vibrations and cyclic loads becomes more and more crucial. Therefore, a reliable mechanical design is essential for high-raise wind turbines to ensure their operating lifetime.

The deterministic assessment of the fatigue damage cannot identify the probability distribution of the fatigue damage. In this work, a probabilistic approach for assessing the probability distribution of the fatigue damage in wind turbine tower is proposed. The normal turbulence model defined in IEC standard is applied to generate the random wind condition.

The probabilistic approach is based on Monte Carlo simulations, which is performed for each mean wind speed varying from the cut-in to cut-out speed and by considering the variance of wind turbulence. The aeroelastic simulation FAST codes [1] is carried out to get the load-time histories used to estimate the cumulative fatigue damage. The Kolmogorov-Smirnov (K-S) test achieves the best-fitting distribution of the fatigue damage. The probabilistic approach developed in this work gives a way to assess the cumulative fatigue damage on the circumference of the cross-section of the wind turbine tower. 


\section{DETERMINISTIC FATIGUE ANALYSIS}

The main process for estimating lifetime of structure builds upon two relationships as shown in Figure 1. The first relationship establishes the correlation between loading environment and stress state (or strain state). This load-stress, or load-strain, relation is usually deduced from finite element method (FEM) or experiments.

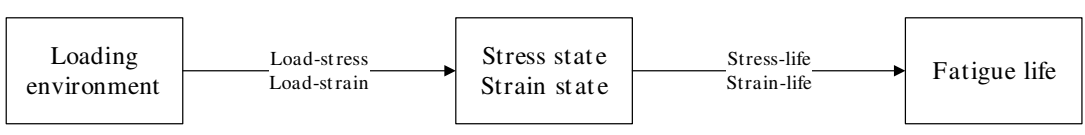

Figure 1: Fatigue life estimation process

No matter which approach described above is applied, a cycle counting algorithm is indispensable to extract a series of fatigue cycles from the complex loading sequences. The most widely applied technique is rainflow counting algorithm proposed by Masanori Matsuishi and Tatsuo Endo [2].

\subsection{Load-stress relation}

In this work, the wind turbine tower is simplified as a thin-walled cylinder structure. Neither the effects of weld and bolt nor the connection components between tower segments are considered. As the result, the stress concentration factor ( $\mathrm{SCF}$ ), i.e., a ratio of the highest stress to a nominal stress, is fixed to 1.0 in the following equation:
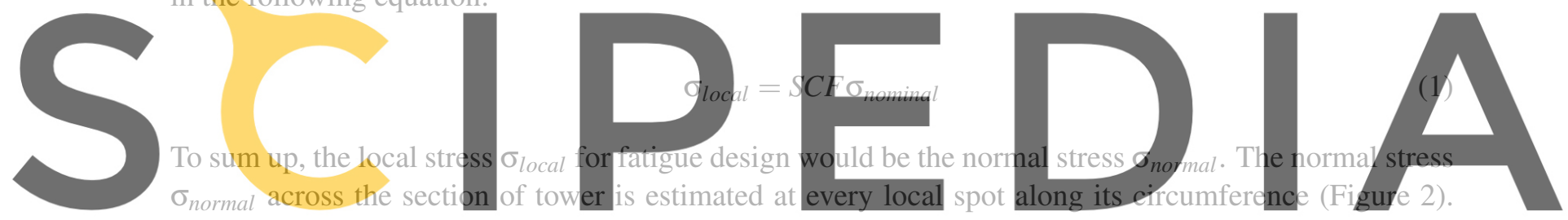

Ignoring the cross section deformation after the loads are exerted, the nominal stress can be computed

Register fby: free at https//www.scipedia.com to download the version without the watermark

$$
\sigma_{\text {local }}(z, t, \alpha)=\frac{F_{z}(z, t)}{A(z)}-\frac{M_{x}(z, t)}{I_{G x}(z)} R(z) \sin \alpha+\frac{M_{y}(z, t)}{I_{G y}(z)} R(z) \cos \alpha
$$

where, $F_{z}$ is axial force, $A$ is nominal cross section area, $M_{x}$ and $M_{y}$ are side-to-side bending moment and fore-after bending moment of tower, respectively, $I_{G x}$ and $I_{G y}$ are second moment of area, $z$ is the vertical distance along $\overrightarrow{z_{t}}$-axis. By evaluating the Equation 2 through all tower gage from $z=0$ to hub height and all local spots from $\alpha=0$ to $2 \pi$, the stress-time history of the whole wind turbine tower is achieved.

\subsection{Stress-life relation}

Fatigue or fatigue damage means the modification of the mechanical properties of materials following repeated application of stress cycles, such applications may lead to the failure of parts. To evaluate such relation, the main approaches involves S-N curve method, mean stress effect and, finally, PalmgrenMiner's rule to get the cumulative fatigue damage.

S-N curve method, also known as Wöhler curve, is commonly used in high-cycle fatigue to characterize 


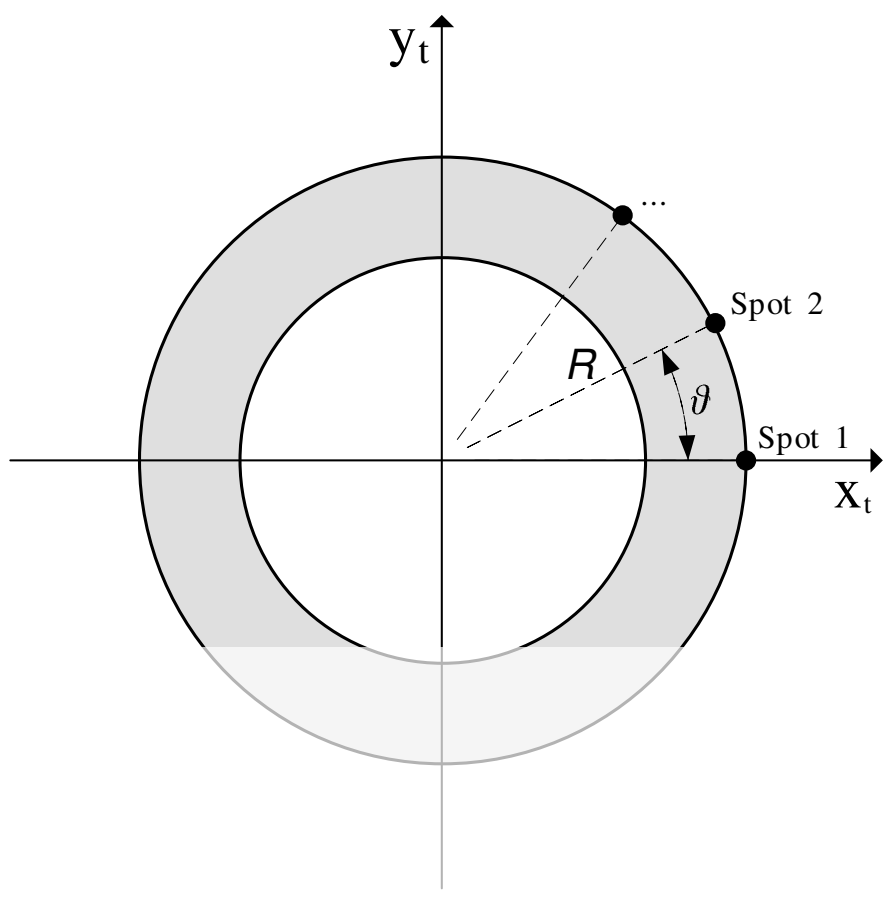

Figure 2: Top view of cross section (gray area) and illustration of spot point
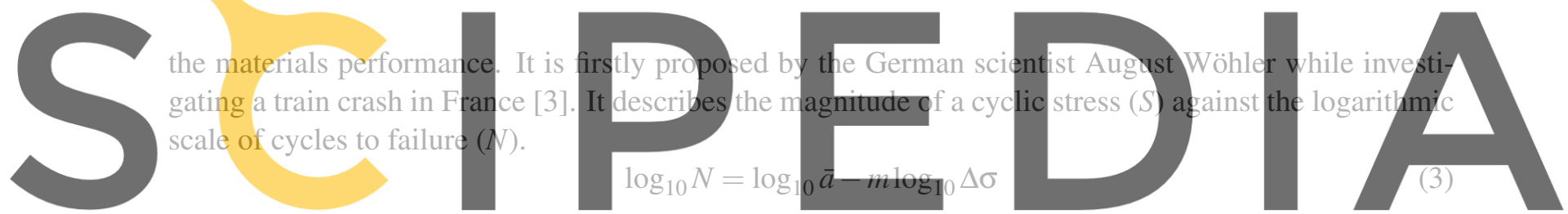

where, $N$ is number of cycles to failure for stress range $\Delta \sigma, \Delta \sigma$ is stress range exported from stress-

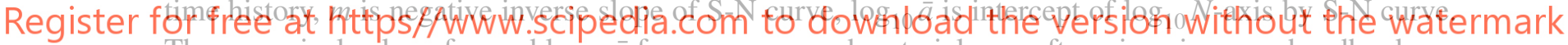

The numerical value of $m$ and $\log _{10} \bar{a}$ for common used materials are often given in many handbooks or

industrial standards [4]. Thus, once the stress range $\Delta \sigma$ is exported, the corresponding number of cycles

to failure $N$ can be easily found by the above equation.

S-N curves characterize the fatigue strength of materials under zero mean stress loads, i.e., $\sigma_{\text {mean }}=0$. However, there are enriched findings in literature that highlight the effect of mean stress $\sigma_{\text {mean }}$ on fatigue behavior of materials $[5,6]$. When the mean stress is small in front of the stress amplitude, the two models are in agreement. However they are no longer in agreement when the mean stress is close to the amplitude. In this case, it is recommended to adopt the most conservative modeling. Thus it can be considered that the Goodman correction is more suitable to model the influence of a mean stress in compression (Equation 4):

$$
\frac{\sigma_{a}}{\sigma_{e}}+\frac{\sigma_{\text {mean }}}{\sigma_{\text {ult }}}=1 \text { with }\left|\sigma_{\text {mean }}\right| \leq \sigma_{\text {ult }}
$$

This model, by using the ultimate strength of material $\sigma_{u l t}$ and the endurance limit $\sigma_{e}$ (also known as fatigue limit), makes it possible to transform each cycle of non-zero mean stress $\sigma_{\text {mean }}$ into an equivalent stress cycle of zero mean with the amplitude $\sigma_{a}$. 
All the corrections made to the data provided by the S-N curves allow these data to be used by the conventional methods in uni-axial fatigue analysis. It is thus possible, for a given amplitude and mean stress cycle, to determine the number of cycles at the end of which the failure will occur. However, for loads composed of different cycles, it becomes necessary to use accumulation laws of fatigue damage.

For stress cycles with amplitude greater than the endurance limit, the damage is irreversible to the structure. The fatiuge damage $D_{m}$, produced by $n_{m}$ cycles with amplitude of $\sigma_{a}$, is related to $N_{m}$, the total number of cycles producing fatigue failure under a stress of constant amplitude $\sigma_{a}$. If $M$ groups of stress cycles with different amplitudes and zero mean stress are considered, then the damage produced by each group is given by Miner's law [7]. Finally, the cumulative fatigue damage $D$ suffered by the structure under all groups of stress cycles is given by the Palmgren formula [8]. To sum up, the Palmgren-Miner linear damge hypothesis is given by:

$$
D=\sum_{i=m}^{M} \frac{n_{m}}{N_{m}}
$$

When the cumulative damage is greater than 1 , failure of structure occurs.

\subsection{Summary}

The fatigue life assessment for wind turbine tower subjected to uni-axial stress involves several steps. The implementation of which in simulation codes is summarized in Figure 3.

As indicated in Figure 1, the first step in estimating fatigue life is to get loading information on structure

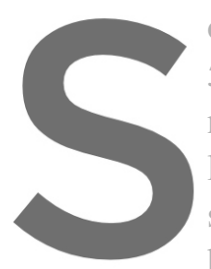
or model. Here in this disse $5 \mathrm{MW}$ reference wind tu no matter what type of

local stress $\sigma_{\text {local }}$ for a giv state is evaluated on each history is computed, the 1oad-stress relation can be

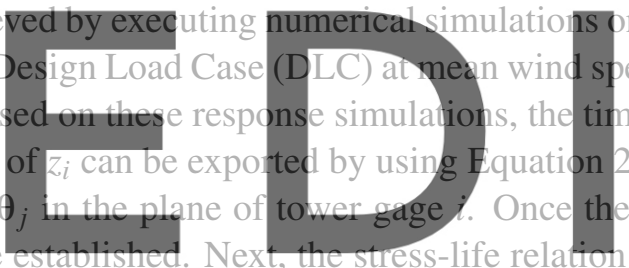
n the NREL
2. The stress
e stre s-rime established and, therefore, the cumulative fatigue damage $D^{(i, j)}$ on each tower gage $i$ and local spot $j$ is Register forrficeeuat https//www.scipedia.com to download the version without the watermark

To conclude, the final set of fatigue damage $\mathbb{D}$ describes naturally the spatial distribution of fatigue loading over the entire wind turbine tower at a given mean wind speed $V_{\text {mean }}$. Since the tower gage $i$ and local spot $j$ are numerically discrete, it is capable to assess the fatigue life at any height from towerbase to tower-top subject to the wind flow coming from any direction between $0^{\circ}$ and $360^{\circ}$. However, this process is deterministic which means that it cannot tell the probability of occurrence of cumulative fatigue damage. This requires the need to assess the fatigue damage in a probabilistic way and hence will be discussed in the next section.

\section{PROBABILISTIC FATIGUE ANALYSIS}

\subsection{Probabilistic concepts}

Due to the random nature of wind, the fatigue life estimation process presented in the previous section must be carried out stochastically. Taking turbulent wind condition for example, the IEC standards require at least six 10-min stochastic simulations, or a continuous 60-min simulation, for each considered mean wind speed [10]. The main idea behinds this approach is that the true response of wind turbine are 


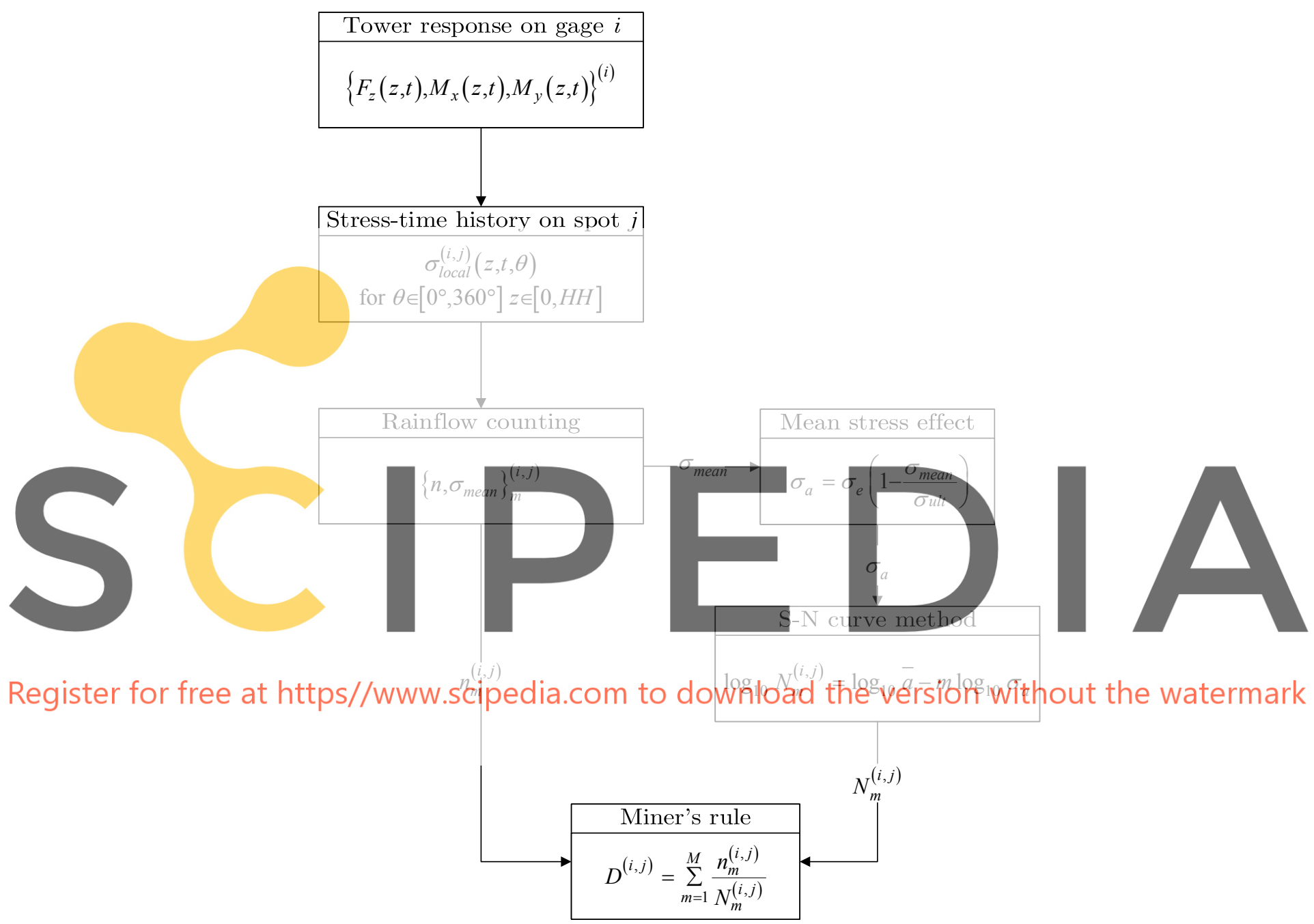

Figure 3: Workflow for assessing cumulative fatigue damage $D$ on tower gage $i$ and local spot $j$ 
based on repeated random sampling and statistical analysis.

This estimation procedure is called Monte Carlo simulation which has been widely applied in many disciplinarians for decades [11]. The principal of Monte Carlo method founds on the law of large numbers which says that the average of the results obtained from a large number of samples should be close to the true value.

After generating sufficient samples, the process described in the last section can predict the cumulative fatigue damage in each sample. In order to conclude a probabilistic distribution of fatigue damage from these samples, a statistical analysis is indispensable including probability distribution fitting and statistical testing.

The distribution fitting is a technique to fit a theoretical probability distribution to a series of empirical data (namely, observations). One of the most dominant methods in distribution fitting is MLE introduced by R.A. Fisher in 1912 [12]. This method determines values for the parameters of probability distribution by maximizing the likelihood function. The likelihood function measures statistically how well a probability distribution fits a set of observations. In other words, it can be considered as a manner to quantify the quality of fitting (also named after goodness of fit) of a distribution on observed values.

By applying the MLE, the values of parameters of both distributions are optimized to give its best fitting on the samples. However, it is hard to tell which distribution describes the observed data better than the other. Since there is no doubt that a close fit distribution leads to good predictions, it is impossible to confidently predict values if the best fit distribution is not reached. In order to choose a closer fit of distribution on the observe data and to ensure more confident predictions, aempare betwe the observed data and the fitted probab be discussed in the next section. One difficulty in distribution can suit the observations goodness of fit of all reference distri
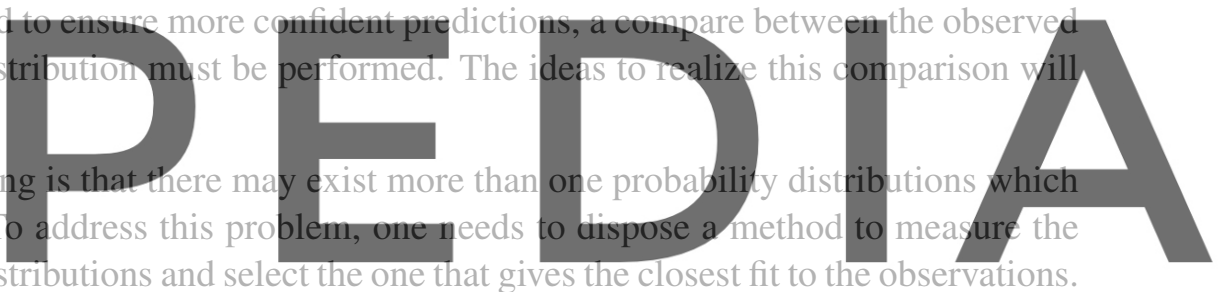

The K-S test proposed by Andrey Nikolaevich Kolmogorov [13] and Nikolaị Vasilyevich Smirnov [14]

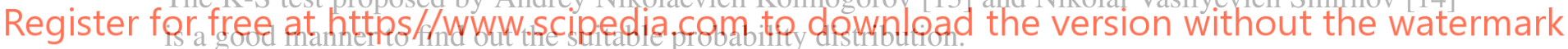

The K-S test is a nonparametric test of the equality for one-dimensional probability distributions. The tested distribution can be either continuous or discontinuous. It can be used to compare a sample to a probability distribution, or to another sample. In principle, the K-S test uses a statistic measuring the distance between the ECDF of the sample and the CDF of the reference distribution. Like other statistical tests, a null hypothesis $\mathbf{H}_{\mathbf{0}}$ is required. In the case of comparing a sample to a reference probability distribution (i.e., one-sample K-S test), the null hypothesis assumes that the observed data is sampled from the reference distribution.

It should be noted that there are many other methods that can also be used to measure the goodness of fit, for instance, Anderson-Darling test [15].

\subsubsection{Probabilistic fatigue assessment}

From the deterministic approach for fatigue assessment mentioned above, the information about cumulative fatigue damage $D^{(i, j)}$ on any tower gage $i$ and local spot $j$ can be deduced. Considering a sample 
of $N$ independent and identically distributed stochastic simulations generated by Monte Carlo method, each simulation requires a numerical modeling in FAST with arbitrary wind condition. Performing the deterministic fatigue analysis on each simulation results in a set of fatigue damage, i.e.,

$$
D_{k}=\left\{D^{(i, j)} \in \mathbb{R}:(i, j) \in \mathbb{N}^{2}\right\} \text { for } k=1, \cdots, N
$$

Next, a pool of reference distributions $\mathcal{P}$ need to be defined by the end-user. It should contain a number of possible distributions to represent the distribution of fatigue damage. For a given position located by tower gage $i$ and local spot $j$, a subset $\mathbf{D}^{(i, j)}$ can be extracted from the sample in which all available data of fatigue damage on this specified position are included, i.e.,

$$
\mathbf{D}^{(i, j)}=\left\{D_{1}^{(i, j)}, \cdots, D_{N}^{(i, j)}\right\} \text { where } \mathbf{D}^{(i, j)} \subset\left\{D_{k} \text { for } k=1, \cdots, N\right\}
$$

According to the MLE distribution fitting process introduced, each distribution in the pool $\mathcal{P}$ will be fitted to the subsample $\mathbf{D}^{(i, j)}$ to get the estimated value $\widehat{\theta}$ for its parameters. Subsequently, the K-S test takes place to compare this fitted distribution $\mathbf{P}(x \mid \widehat{\theta})$ with the same subsample $\mathbf{D}^{(i, j)}$. The K-S statistic $D_{n}^{(i, j)}$ resulted from this comparison is used to represent the goodness of fit for this estimated distribution, i.e.,

$$
\mathbf{P}(x \mid \widehat{\theta}) \mapsto D_{n}^{(i, j)}
$$

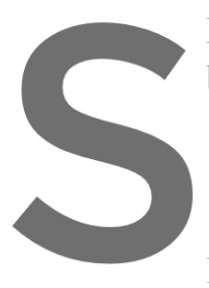

Finally, the distribution

bution of cumulative fati

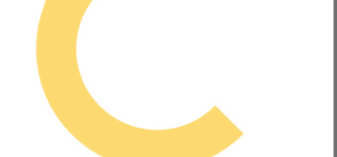

It should be noted that the process
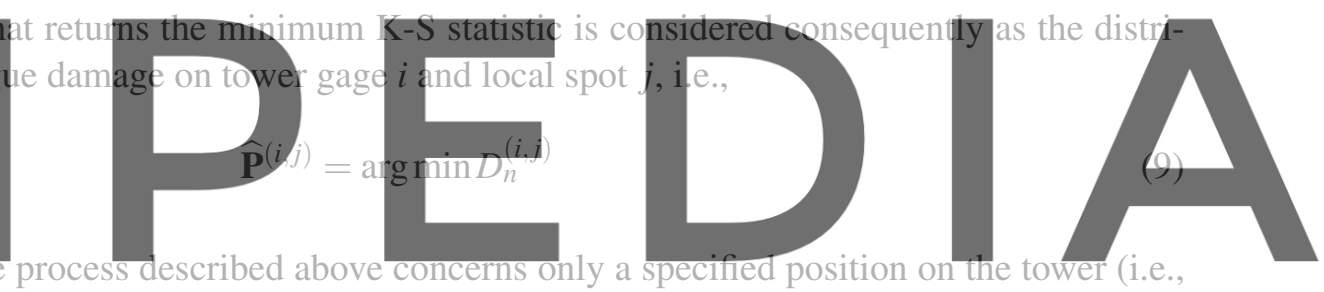

tower gage $i$ and local spot $j$ ). Repeating such process for all positions outlines a probability profile

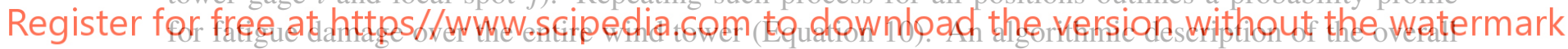

process is detailed in Algorithm 1.

$$
\widehat{\mathcal{P}}=\left\{\widehat{\mathbf{P}}^{(i, j)} \in \mathcal{P}:(i, j) \in \mathbb{N}^{2}\right\}
$$

\section{APPLICATION}

The design load case of power production with normal turbulence model (DLC1.2) issued from the IEC 61400-1 standard specifies a scenario for generating electrical power in the face of wind considering Normal Turbulence Model (NTM). The state of wind turbine is considered as in normal production without technical fault or undesired incident. The wind velocities in three directions $\left(\vec{x}_{t}, \vec{y}_{t}\right.$ and $\left.\vec{z}_{t}\right)$ are unsteady and randomly changed during a period of time. The IEC specifications suggest either a period of 10-min with at least six numerical simulations or a full simulation of 60-min. For convenience of running, the 10-min period is chosen by the author as the simulated time in each run. The number of runs depends on the number of observations in the sample. To ensure enough data for probabilistic fatigue analysis, a number of 10000 observations is planned for each case of mean wind speed. Each 


\section{Algorithm 1: Probabilistic fatigue assessment on wind turbine tower \\ Generate a sample $\mathcal{D}$ of $N$ independent and identically distributed observations using Monte Carlo simulation \\ foreach observation $k$ in $\mathcal{D}$ do}

Run a numerical simulation in FAST under an arbitrary wind condition

Execute the deterministic fatigue analysis on this simulation and return the cumulative fatigue damage on all positions of wind turbine tower, i.e., $D_{k}=\left\{D^{(i, j)} \in \mathbb{R}:(i, j) \in \mathbb{N}^{2}\right\}$

\section{end foreach}

Define a pool of reference distributions $\mathcal{P}$

foreach tower gage $i$ do

foreach local spot j do
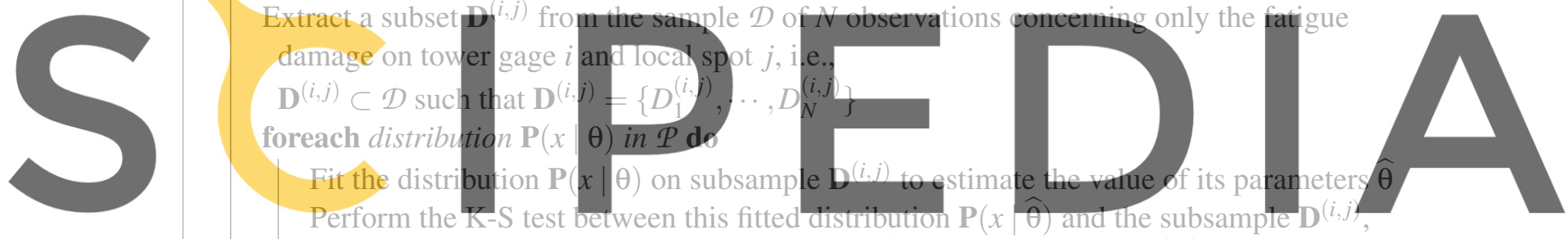

Register for free at fottpa//WWW.Scipedia.com to download" the version without the watermark

Select the distribution that has the minimum K-S statistic among all fitted distributions, i.e.,

$$
\widehat{\mathbf{P}}=\arg \min D_{n}^{(i, j)}
$$

Add this distribution to the set $\widehat{\mathcal{P}}=\left\{\widehat{\mathbf{P}}^{(i, j)} \in \mathcal{P}: \widehat{\mathbf{P}}^{(i, j)}=\widehat{\mathbf{P}}\right\}$

end foreach

end foreach 
Table 1: Summary of DLC1.2

\begin{tabular}{ll}
\hline DLC1.2 & Power production \\
\hline Description & Simulation of power production in turbulent wind condition \\
\hline \multirow{3}{*}{ Simulation set-up } & Mean wind speed: $\left[V_{\text {in }}, V_{\text {in }}+1, \cdots, V_{\text {out }}\right], 23$ cases in total \\
& Turbulence: Normal Turbulence Model (NTM) \\
& Simuliated time: $600 \mathrm{~s}$ \\
\hline \multirow{3}{*}{ Comment } & A number of 10000 simulations is planned for each case of mean wind speed. \\
& This leads to a sum of 230000 runs in FAST simulation codes \\
& As for the post-processing, each run performs the probabilistic fatigue analysis \\
& on 11 tower gages, each of them contains 36 equally separated local spots, that \\
& is, a total of $11 \times 36=396$ local spots. \\
& The deterministic fatigue assessment follows the guidelines given in the DNVGL- \\
& RP-C203 standard [4].
\end{tabular}

observation implies a unique random seed number that will be used to generate a unique NTM wind condition in FAST codes.

By definition, the mean wind speed $V_{\text {mean }}$ at hub height in NTM varies from $V_{\text {cut }- \text { in }}=3 \mathrm{~m} / \mathrm{s}$ to $V_{\text {cut }}$-out $=$ $25 \mathrm{~m} / \mathrm{s}$ at step of $1 \mathrm{~m} / \mathrm{s}$, that is, a total of 23 cases of mean wind speed. Table 1 summarizes all the
characteristics of DLC1.2.
During post-processing of each simulation, phechanic responses for all tower gages are exported. Each
tower gage is equally divided into 36 loeal spots. Thus, a total of $11 \times 36=396$ local spots will be used
to take place the probabilistic fatgue analysis for the wind tower. Each of them will have 23 individual probability distributions of 10 -min cumulative fatigue damage corresponding respectively to the 23 cases

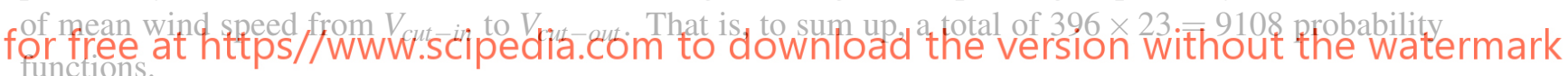

In order to carry out fatigue analysis in numerical environment, the engineering guidance from the recommended practice DNVGL-RP-C203 [4] is applied. The carbon manganese steel material (C-Mn) is considered in the simulation. The S-N curve of class B1 in air environment is assumed true which has an endurance limit of $\sigma_{e}=106.97 \mathrm{MPa}$ at $10^{7}$ cycles.

\subsection{Result and discussion}

Figure 4 plots the data in radar chart (also known as Kiviat diagram [16]). The radial axis, or polar axis, represents the 10-min cumulative fatigue damage on which the zero point, i.e., zero damage, is centered in the chart. The interior perimeters indicate the increment on fatigue damage from zero (center) to its maximum (exterior perimeter). On the outside of the exterior perimeter locates the azimuth angle $\alpha$ of local spots in degrees. By convention, the local 1 places on the right side of the cross section and has an azimuth angle $\alpha=0^{\circ}$ (Figure 2). The colored lines inside the radar graph stand for the different percentiles denoted as $P_{i}$ where $i$ means $i$-th percentile. For example, $P_{5}$ (the blue dotted line) is the 5 th percentile below which $5 \%$ of the observations may be found (the gray surface in Figure 4). In other 


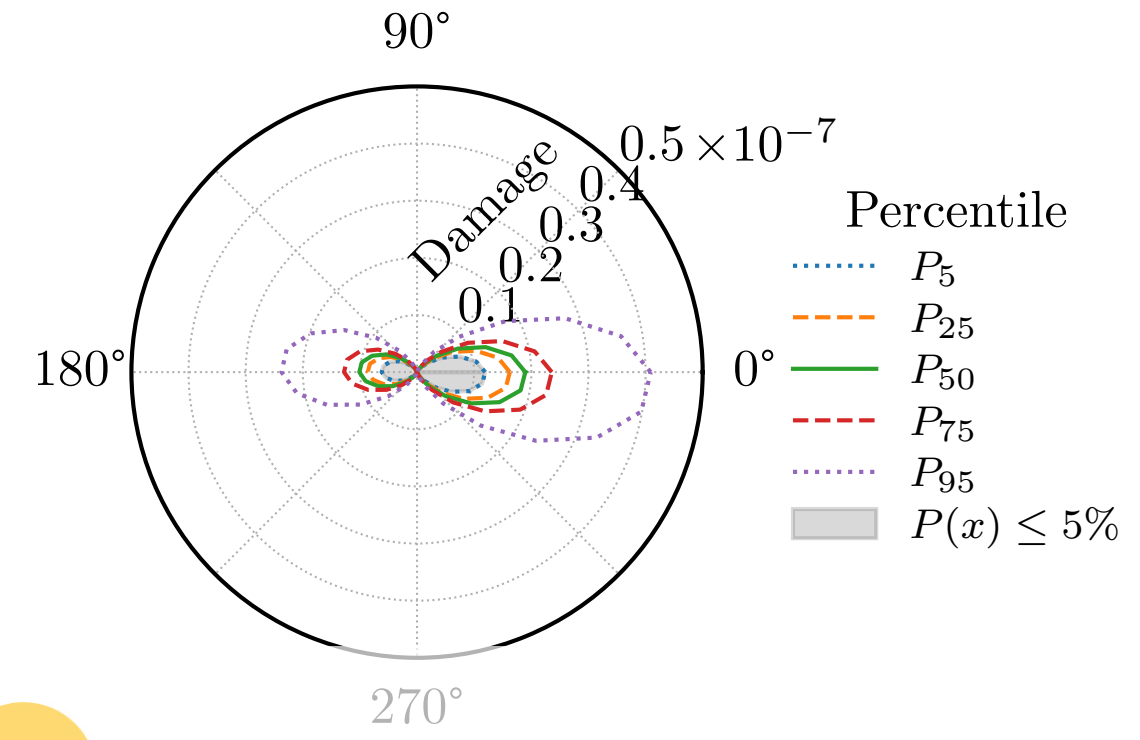

Figure 4: DLC1.2 distribution of 10-min cumulative fatigue damage on tower gage 1 radar map (cylindrical coordinate system)

words, the wind turbine tower has likely a chance of $5 \%$ to subject a fatigue damage value inside the gray surface. More percentiles such as $P_{25}, P_{50}, P_{75}$ and $P_{95}$ are highlighted by orange dashed line, green line, red dashed line and purple dotted line respectively in Figure 4. $P_{50}$ as the second quartile

5 CONCLUSION

In summary, the proposed framework offers a nove
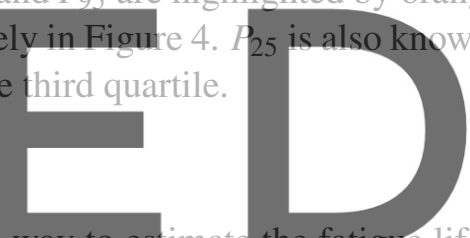

tower. It extends the fatigue damage assessment from deterministic process to probabilistic process. The

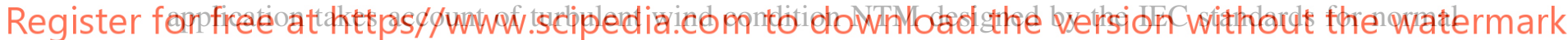
power production. The cumulative fatigue damage is assessed under all mean wind speed in the operating range of $\left[V_{\text {in }}, V_{\text {out }}\right]$ through all tower gages and local spots. By means of this analysis, a survey of the fatigue damage accumulated over the wind turbine tower is acquired. Since the final profile of fatigue damage is based on statistics and probability, it opens the gate to carry out many statistical analysis and probability based analysis such as Reliability Based Design Optimization.

\section{REFERENCES}

[1] Jason M. Jonkman and Marshall L. Buhl Jr. FAST User's Guide. Tech. rep. National Renewable Energy Laboratory (NREL), 2005, p. 143.

[2] Masanori Matsuishi and Tatsuo Endo. "Fatigue of metals subjected to varying stress". In: Japan Society of Mechanical Engineers, Fukuoka, Japan 68.2 (1968), pp. 37-40.

[3] August Wöhler. "Versuche zur Ermittlung der auf die Eisenbahnwagenachsen einwirkenden Kräfte und die Widerstandsfähigkeit der Wagen-Achsen". In: Zeitschrift für Bauwesen 10.1860 (1860), pp. 583-614.

[4] DNV GL AS. Recommended Practice - Fatigue Design of Offshore Steel Structures. 2019. 
[5] J.R. Rice and Dennis Michael Tracey. "On the ductile enlargement of voids in triaxial stress fields". In: Journal of the Mechanics and Physics of Solids 17.3 (1969), pp. 201-217.

[6] Arthur L Gurson. "Continuum theory of ductile rupture by void nucleation and growth: Part I-Yield criteria and flow rules for porous ductile media". In: Journal of engineering materials and technology 99.1 (1977), pp. 2-15.

[7] M A Miner. "Cumulative fatigue damage". In: Journal of applied mechanics 12.3 (1945), A159A164.

[8] Arvid Palmgren. "Die lebensdauer von kugellargern". In: Zeitshrift des Vereines Duetsher Ingenieure 68.4 (1924), p. 339.

[9] Jason M. Jonkman et al. Definition of a 5-MW Reference Wind Turbine for Offshore System Development. Tech. rep. February. National Renewable Energy Laboratory (NREL), 2009. DOI: 10 . $2172 / 947422$.

[10] International Electrotechnical Commission (IEC). Wind turbines - Part1: Design requirements. 2005.

[11] Metodi Mazhdrakov, Dobriyan Benov, and Nikolai Valkanov. The Monte Carlo Method: Engineering Applications. ACMO Academic Press, 2018.

[12] John Aldrich. "RA Fisher and the making of maximum likelihood 1912-1922". In: Statistical science 12.3 (1997), pp. 162-176.

[13] Andrey Kolmogorov. "Sulla determinazione empirica di una lgge di distribuzione". In: Inst. Ital. Attuari, Giorn. 4 (1933), pp. 83-91.

[14] Nickolay Smirnov. "Table for estimating the goodness of fit of empirical distributions". In: The annals of mathematical statistics 19.2 (1948), pp. 279-281.

[15] Theodore W Anderson and Donald A Darling. "Asymptotic theory of certain" goodness of fit" criteria based on stochastic processes". In: The annals of mathematical statistics (1952), pp. 193212.

[16] Kenneth W Kolence and Philip J Kiviat. "Software unit profiles \& Kiviat figures". In: ACM SIGMETRICS performance evaluation review 2.3 (1973), pp. 2-12. 